\title{
On a Consumer Problem
}

\section{Sabir Isa Hamidov}

Mathematical-Cybernetics Department, Baku State University, Baku, Azerbaijan

\section{Email address:}

Sabir818@yahoo.com

\section{To cite this article:}

Sabir Isa Hamidov. On a Consumer Problem. Pure and Applied Mathematics Journal. Vol. 5, No. 6, 2016, pp. $205-210$.

doi: 10.11648/j.pamj.20160506.15

Received: February 10, 2015; Accepted: October 20, 2016; Published: November 15, 2016

Abstract: The model of economic dynamics of the Leontief type is considered. The problem of determining the equilibrium state of the model with a fixed budget is investigated. It is proved that the state of equilibrium exists, if the trajectory model is a solution to the consumer problem.

Keywords: Production Mapping, Utility Function, Equilibrium

\section{Introduction}

Let at the moment $t$ the production mapping $a[1,4]$ be given

$$
\begin{gathered}
a(X)=\left\{\tilde{X}=\left(\tilde{x}^{1}, \ldots, \tilde{x}^{n \cdot}\right) \in\left(R_{+}^{n}\right)^{n} \mid 0 \leq \sum_{i=1}^{n} \tilde{x}^{i \cdot} \leq(1)\right. \\
\leq \sum_{i=1}^{n} B^{k} \cdot x^{k^{\cdot}}+\left(F^{1}\left(x^{1 \cdot}\right), \ldots, F^{n}\left(x^{n \cdot}\right)\right), x^{k \cdot} \\
\left.=\left(x^{k 1}, \ldots, x^{k n}\right), k=\overline{1, n}\right\},
\end{gathered}
$$

where $X=\left(x^{1 \cdot}, \ldots, x^{n \cdot}\right) \in\left(R_{+}^{n}\right)^{n}, B^{k}$ is a diagonal matrix the main diagonal of which has a form

$$
\left(v^{k 1}, \ldots, v^{k n}\right), v^{k i} \in[0,1](k, i=\overline{1, n}) ;
$$

$F^{j}(x)$ are production functions of the branches:

$$
F^{j}(x)=\min _{i=\overline{1, n}} \frac{x^{i}}{c^{i j}}, c^{i j}>0(i, j=\overline{1, n}) .
$$

Production mapping $a^{k}(x)$ [4] of the branch $k$ has a form:

$$
a^{k}(x)=\left\langle 0, B^{k} x+\left(0, \ldots, 0, F^{k}(x), 0, \ldots, 0\right)\right\rangle\left(x \in R_{+}^{n}\right) .
$$

Note. The mapping $a^{k}$ is completely defined by the set

$$
\left\{v^{k i}, c^{i k},(i, k=\overline{1, n})\right\}
$$

where $c^{i k}>0, v^{k i} \in[0,1](k, i=\overline{1, n})$.

Let

$$
I=\{1,2, \ldots, n\}, \ell=\left(\ell^{1}, \ldots, \ell^{n}\right) .
$$

If consider (2) then the utility function of the $k-t h$ branch takes the form [2]

$$
U^{k}\left(\ell, x^{k}\right)=\sum_{j \in I} \ell^{j} \cdot v^{k j} \cdot x^{k j}+\ell^{k} \cdot \min _{j \in I} \frac{x^{k j}}{c^{j k}}
$$

where $\ell=\left(\ell^{1}, \ell^{2}, \ldots, \ell^{n}\right)$ is a cost vector, the set $I$ is defined by the formula (5).

By the definition the set $\left(P, x^{1}, \ldots, x^{n}, y\right)$ is an equilibrium state if $\sum_{k \in I} x^{k \cdot}=y$ and $x^{k \cdot}$ is a solution of the $k-t h$ consumer problem [5-7]

$U^{k}(\ell, x) \rightarrow \max$, subject to $[P, x] \leq \lambda^{k}, x \in R_{+}^{n}(k \in I)$,

where $U^{k}$ is in the form (6), $\lambda^{k}$ is a component of the budget vector $\Psi=\left(\lambda^{1}, \ldots, \lambda^{n}\right)$.

Let the vector $\bar{x}^{k}$ be a solution of the $k-t h$ consumer problem

$$
U^{k}(\ell, x) \rightarrow \max , x \in \tilde{V}=\{x \geq 0,[P, x] \leq 1\}(k \in I) .
$$

Then the equilibrium vector $x^{k \cdot}$ has a form [4]

$$
x^{k \cdot}=\lambda^{k} \cdot \bar{x}^{k \cdot}(k \in I) .
$$

Note that from (4) and (9) follows that

$$
y=\sum_{k \in I} x^{k}=\sum_{k \in I} \lambda^{k} \cdot \bar{x}^{k} .
$$


This means that

$$
y \in \operatorname{cone}\left\{\bar{x}^{k \cdot} \mid k \in I\right\} .
$$

In the future, we will be interested in the following problem. Given a mapping $a^{k}$, i.e. $v^{k i}, c^{i k}(k, i \in I)$ and $\left(P, x^{1}, \ldots, x^{n}, y\right)$ are a set of the vectors such that $\sum_{i \in I} x^{i \cdot}=$ $y, x^{i \cdot} \leq 0$. Determine whether there is model $\mathrm{M}$ with given $v^{k i}, c^{i k}$ in which the $\operatorname{set}\left(P, x^{1}, \ldots, x^{n}, y\right)$ is an equilibrium, and, if so, to find it, that is, specify $\ell^{i}$ and $\lambda^{i}(i \in I)$ such that this set is a state of equilibrium in the model $\{y, U(\ell), \Psi\}$. From this problem, it follows that it is a problem with $2 \cdot n$ unknowns $\ell^{i}, \lambda^{i}(i \in I)$.

\section{Materials and Methods}

Let $x \in R_{+}^{n}$. Throughout the following notation will be used below

$$
\begin{gathered}
I_{1}(x)=\left\{i \in I \mid x^{i}=0\right\}, \\
I_{2}(x)=\left\{i \in I \mid x^{i}>0\right\}, \\
R^{k}(x)=\left\{i \in I \mid \frac{x^{i}}{c^{i k}}=\min _{j \in I} \frac{x^{j}}{c^{j k}}\right\}(k \in I), \\
Q^{k}(x)=I \backslash R^{k}(x)(k \in I) .
\end{gathered}
$$

Before talking about equilibrium, we examine the consumer problem. Along with set $\tilde{V}$ in the consumer problem (8) we can consider the set

$$
V=\{x \geq 0 \mid[P, x]=1\} .
$$

Due the homogeneity of the functions $U^{k}(\ell, x)$ their maximums on the sets $\tilde{V}$ and $V$ coincide.

Let $\bar{x}$ be a maximum point in the $k-t h$ consumer problem (8). Then this point satisfies the necessary and sufficient conditions for an extremum differentiable on the direction function

$$
\left(U^{k}\right)^{\prime}(\bar{x}, g) \leq 0 \forall g \in G_{\bar{x}}(V),
$$

where [9]

$G_{\bar{x}}(V)=\left\{g \in R^{n} \mid \exists \alpha_{o}>0: \bar{x}+\alpha \cdot g \in V \forall \alpha \in\left(0, \alpha_{o}\right)\right\}$,

And the set $V$ is defined by the formula (11).

Introduce the set $G_{\bar{x}}(V)$ :

$$
\begin{gathered}
G_{\bar{x}}(V)=\left\{g \in R^{n} \mid \exists \alpha_{o}>0:[P, \bar{x}+\alpha \cdot g]=1, \bar{x}+\alpha \cdot g\right. \\
\left.\geq 0 \forall \alpha \in\left(0, \alpha_{o}\right)\right\} .
\end{gathered}
$$

Since $[P, \bar{x}]=1$ then from $[P, \bar{x}+\alpha \cdot g]=[P, \bar{x}]+\alpha$. $[P, g]=1$ follows that $[P, g]=0$. From the condition $\bar{x}+\alpha \cdot g \geq 0$ or $\bar{x}^{i}+\alpha \cdot g^{i} \geq 0$ for $\forall i \in I$ follows that

a) If $\bar{x}^{i}=0$, then $\alpha \cdot g^{i} \geq 0$, consequently $g^{i} \geq 0$;

b) If $\bar{x}^{i}>0$ then $\bar{x}^{i}+\alpha \cdot g^{i} \geq 0$ for $\forall g^{i}$ small enough $\alpha$.

From the foregoing, we find that the set $G_{\bar{x}}(V)$ can be written as

$$
G_{\bar{x}}(V)=\left\{g \in R^{n} \mid[P, g]=0, g^{i} \geq 0 \forall i \in I_{1}(\bar{x})\right\},
$$

where the set $I_{1}(\bar{x})$ is defined by the formula (10).

Let us solve the properties of the solution of the consumer problem. Particular attention is paid to how these properties are associated with the structure of the set $I_{1}(\bar{x})$.

Lemma 1. Let $\bar{x}$ be a solution of the $k-t h(k \in I)$ consumer problem. Then if $I_{1}(\bar{x}) \neq \emptyset$ then $I_{1}(\bar{x}) \subset R^{k}(\bar{x})$.

Proof. Let $\bar{x}$ be a seeking solution of the $k-t h$ consumer problem and $I_{1}(\bar{x}) \neq \varnothing$, i.e. there exists an index $i \in I$ such that $x^{i}=0$. Then suppose that $R^{k}(\bar{x})=I$. But since $I_{1}(\bar{x}) \neq$ $\emptyset$ we get $\bar{x}=0$ that is impossible. If $I_{1}(\bar{x}) \subset Q^{k}(\bar{x})$, then $\bar{x}^{i}<0$ for all $i \in R^{k}(\bar{x})$ that is also impossible. Consequently, $I_{1}(\bar{x}) \subset R^{k}(\bar{x})$.

The lemma is proved.

Consequence. If $R^{k}(\bar{x})=I$ then $I_{1}(\bar{x}) \neq \emptyset$.

Let us study in detail the $k$-th consumer problem. Let $\bar{x}$ be a solution of this problem and the vector $P=\left(P^{1}, \ldots, P^{n}\right)$ be given where $P^{i}>0$ for any $i \in I$.

The utility function of the $k$-th branch in the point $x$ has a form [2]

$$
U^{k}(\ell, x)=\sum_{j \in I} \ell^{j} \cdot v^{k j} \cdot x^{j}+\ell^{k} \cdot \min _{j \in I} \frac{x^{j}}{c^{j k}}(k \in I),
$$

where $\ell=\left(\ell^{1}, \ldots, \ell^{n}\right)$ is a given cost vector.

Introduce the vector

$$
\ell_{v}^{k}=\left(\ell^{1} \cdot v^{k 1}, \ldots, \ell^{n} \cdot v^{k n}\right)(k \in I) .
$$

Then (14) takes the form

$$
U^{k}(\bar{x})=U^{k}(\ell, \bar{x})=\left[\ell_{v}^{k}, \bar{x}\right]+\ell^{k} \cdot \min _{j \in I} \frac{\bar{x}^{j}}{c^{j k}}(k \in I) .
$$

To investigate the $k$-th consumer problem, we apply the necessary and sufficient conditions for the extremum, according which the maximum is reached in the point $\bar{x}$ if and only if

$$
\left(U^{k}\right)^{\prime}(\bar{x}, g) \leq 0 \forall g \in G_{\bar{x}}(V)(k \in I),
$$

where the cone $G_{\bar{x}}(V)$ is defined by the formula (13).

It is well known that $\left(U^{k}\right)^{\prime}(\bar{x}, g)=q^{k}(g)$, where

$$
q^{k}(g)=\left[\ell_{v}^{k}, g\right]+\ell^{k} \cdot \min _{j \in R^{k}(\bar{x})} \frac{g^{j}}{c^{j k}}, g \in R^{n} .
$$

Introduce the denotations

$$
\tilde{q}^{k}(g)=\ell^{k} \cdot \min _{j \in R^{k}(\bar{x})} \frac{g^{j}}{c^{j k}}(k \in I) .
$$

Thus if in the point $\bar{x}$ the maximum is reached then

$$
\begin{aligned}
q^{k}(g) \leq 0 \forall g \in G_{\bar{x}}(V) & =\left\{g \in R^{n} \mid[P, g]=0, g^{i} \geq\right. \\
\left.0 \forall i \in I_{1}(\bar{x})\right\}, & (17)
\end{aligned}
$$

where the set is defined $I_{1}(\bar{x})$ by $(10)$.

Consider some particular cases.

1. Let

$$
I_{1}(\bar{x})=\emptyset,
$$

where $\bar{x}$ is a maximum pint in the $k$-th consumer problem and the set $I_{1}(x)$ is defined by the formula (10). 
In this case from (13) follows that $G_{\bar{x}}(V)=\Omega$, where

$$
\Omega=\left\{g \in R^{n} \mid[P, g]=0\right\} .
$$

Then the necessary and sufficient condition for the optimality $\bar{x}$ in the branch $k$ takes the form

$$
q^{k}(g)=\left[\ell_{v}^{k}, g\right]+\tilde{q}^{k}(g) \leq 0, \forall g \in \Omega,
$$

where the function $\tilde{q}^{k}(g)$ is defined by the formula (16).

Lemma 2. The following conditions are equivalent:

1) $q^{k}(g) \leq 0, \forall g \in \Omega$

2) $\exists \mu^{k}>0: \mu^{k} \cdot P \in \partial q^{k}$.

where $\partial q^{k}$-is a superdifferential of the function $q^{k}$.

Proof. The function $q^{k}(g)$ is concave. Let the inequality $q^{k}(g) \leq 0 \forall g \in \Omega$ take place.

Since $q^{k}(0)=0$ then in the point $\bar{g}=0$ the function $q^{k}$ reaches its maximum in the set $\Omega$. It is well known that necessary and sufficient conditions for the maximum of the concave function $q^{k}(g)$ in the point $\bar{g}=0$ on the set

$$
\left\{g \in R^{n} \mid[P, g]=0\right\}
$$

Consist in the existing of the element $f^{k} \in \partial q^{k}(\bar{g})$ such that

$$
\max _{[P, g]=0}\left[f^{k}, g\right]=0 .
$$

But it means that $\left[f^{k}, g\right]=0$ in the same place where $[P, g]=0$. It implies that for some $\mu^{k}$ takes place the following equality

$$
f^{k}=\mu^{k} \cdot P .
$$

Since $f^{k}$ and $P$ are positive we get $\mu^{k}>0$.

Citing the same arguments, but in reverse order, it is easy to show that from condition 2) of the lemma follows condition 1).

The proof is complete.

Lemma 3.Superdifferential of the function $q^{k}(g)(g \in$ $R^{n}$ ) defined by the formula (15) has a form [8-10]

$$
\partial q^{k}=\ell_{v}^{k}+\partial \tilde{q}^{k}(k \in I),
$$

where

$$
\begin{gathered}
\ell_{v}^{k}=\left(\ell^{1} \cdot v^{k 1}, \ldots, \ell^{n} \cdot v^{k n}\right), \\
\tilde{q}^{k}(g)=\ell^{k} \cdot \min _{i \in R^{k}(\bar{x})} \frac{g^{i}}{c^{i k^{\prime}}}
\end{gathered}
$$

moreover

$$
\begin{aligned}
\partial \tilde{q}^{k}=\left\{f=\ell^{k}\right. & \cdot\left(f^{1}, \ldots, f^{n}\right) \mid \exists \alpha^{i} \geq 0: \sum_{i \in R^{k}(\bar{x})} \alpha^{i}=1, f^{i} \\
= & \frac{\alpha^{i}}{c^{i k}}
\end{aligned}
$$$$
\left.i \in R^{k}(\bar{x}) ; f^{i}=0, i \in Q^{k}(\bar{x})\right\}(k \in I) .(21)
$$

Proof. From (15), (16) we have

$$
\partial q^{k}=\ell_{v}^{k}+\partial \tilde{q}^{k}(k \in I) .
$$

Define the vector $C_{i}^{k}=\frac{1}{c^{i k}} \cdot e^{i} \in R^{n}$, where $e^{i}$ is $i-t h$ coordinate ort $(i, k \in I)$. Then

$$
\left[C_{i}^{k}, g\right]=\frac{g^{i}}{c^{i k}}(i \in I)
$$

and

$$
\tilde{q}^{k}(g)=\ell^{k} \cdot \min _{i \in I}\left[C_{i}^{k}, g\right]\left(g \in R^{n}\right) .
$$

From the definition of the superdifferntial we obtain (21). The lemma is proved.

Lemma 4. The number $\mu^{k}(k \in I)$ defined in the Lemma 2. Is equal to

$$
\mu^{k}=\frac{\ell^{k}+\sum_{i \in R^{k}(\bar{x})} \ell^{i \cdot \cdot v^{k i} \cdot c^{i k}}}{\sum_{i \in R^{k}(\bar{x})} P^{i \cdot} \cdot c^{i k}}(k \in I)
$$

where $\ell_{v}^{k}=\left(\ell^{1} \cdot v^{k 1}, \ell^{2} \cdot v^{k 2}, \ldots, \ell^{n} \cdot v^{k n}\right)$.

Proof. Let $\mu^{k}>0$ be such that $\mu^{k} \cdot P \in \ell_{v}^{k}+\partial \tilde{q}^{k}$, i.e. condition 2) of the Lemma 2 is satisfied. Using (21) one can obtain from this that for some $\alpha^{i} \geq 0, \sum_{i \in R^{k}(\bar{x})} \alpha^{i}=1$ the following equality holds true

$$
\left\{\begin{array}{c}
\mu^{k} \cdot P^{1}=\ell^{1} \cdot v^{k 1}+\frac{\alpha^{1}}{c^{1 k}} \cdot \ell^{k} \\
\cdots \cdots \cdots \cdots \cdots \cdots \cdots \cdots \cdots \cdots \cdots \cdots \cdots \cdots \cdots \cdots \cdots \cdots \cdots \cdots \cdots \cdots \cdots \cdots \cdots \cdots \cdots \cdots \\
\mu^{k} \cdot P^{r}=\ell^{r} \cdot v^{k r}+\frac{\alpha^{r}}{c^{r k}} \cdot \ell^{k} \\
\mu^{k} \cdot P^{r+1}=\ell^{r+1} \cdot v^{k r+1} \\
\cdots \cdots \cdots \cdots \cdots \cdots \cdots \cdots \cdots \\
\mu^{k} \cdot P^{n}=\ell^{n} \cdot v^{k n}
\end{array}\right.
$$

where $r=\left|R^{k}(\bar{x})\right|$.

It follows from the last that

$$
\begin{aligned}
\mu^{k}= & \frac{\ell^{1} \cdot v^{k 1} \cdot c^{1 k}}{P^{1} \cdot c^{1 k}}+\frac{\alpha^{1} \cdot \ell^{k}}{P^{1} \cdot c^{1 k}}=\cdots \\
& =\frac{\ell^{r} \cdot v^{k r} \cdot c^{r k}}{P^{r} \cdot c^{r k}}+\frac{\alpha^{r} \cdot \ell^{k}}{P^{r} \cdot c^{r k}}= \\
& =\frac{\ell^{r+1} \cdot v^{k r+1}}{P^{r+1}}=\cdots=\frac{\ell^{n} \cdot v^{k n}}{P^{n}} .
\end{aligned}
$$

Let's fix the index $j \in R^{k}(\bar{x})$ and express all $\alpha^{i}(i \in$ $\left.R^{k}(\bar{x}) \backslash\{j\}\right)$ through $\alpha^{j}$ :

$$
\begin{gathered}
\alpha^{i}=\frac{1}{\ell^{k}}\left(\frac{\alpha^{j} \cdot \ell^{k}}{P^{j} \cdot c^{j k}} \cdot P^{i} \cdot c^{i k}+\frac{\ell^{j} \cdot v^{k j} \cdot c^{j k}}{P^{j} \cdot c^{j k}} \cdot P^{i} \cdot c^{i k}-\ell^{i} \cdot v^{k i} \cdot c^{i k}\right) \\
\forall i \in R^{k}(\bar{x}) \backslash\{j\} .
\end{gathered}
$$

Due the conditions $\sum_{i \in R^{k}(\bar{x})} \alpha^{i}=1$

$$
\sum_{i \in R^{k}(\bar{x})} \alpha^{i}=\frac{\alpha^{j}}{P^{j} \cdot c^{j k}} \cdot \sum_{i \in R^{k}(\bar{x}) \backslash\{j\}} P^{i} \cdot c^{i k}+\alpha^{j}+
$$




$$
\begin{gathered}
+\frac{\ell^{j} \cdot v^{k j} \cdot c^{j k}}{\ell^{k} \cdot P^{j} \cdot c^{j k}} \cdot \sum_{i \in R^{k}(\bar{x})} P^{i} \cdot c^{i k}-\frac{1}{\ell^{k}} \cdot \sum_{i \in R^{k}(\bar{x}) \backslash\{j\}} \ell^{i} \cdot v^{k i} \cdot c^{i k}= \\
=\frac{\alpha^{j} \cdot \ell^{k} \cdot \sum_{i \in R^{k}(\bar{x})} P^{i} \cdot c^{i k}+\ell^{j} \cdot v^{k j} \cdot c^{j k} \cdot \sum_{i \in R^{k}(\bar{x})} P^{i} \cdot c^{i k}}{\ell^{k} \cdot P^{j} \cdot c^{j k}}- \\
-\frac{P^{j} \cdot c^{j k} \cdot \sum_{i \in R^{k}(\bar{x})} \ell^{i} \cdot v^{k i} \cdot c^{i k}}{\ell^{k} \cdot P^{j} \cdot c^{j k}}=1 .
\end{gathered}
$$

From this we obtain $\alpha^{j}\left(j \in R^{k}(\bar{x})\right)$ :

$$
\alpha^{j}=\frac{\ell^{k}+\sum_{i \in R^{k}(\bar{x})} e^{i} \cdot v^{k i} \cdot c^{i k}}{\ell^{k} \cdot \sum_{i \in R^{k}(\bar{x})} P^{i} \cdot c^{i k}} \cdot P^{j} \cdot c^{j k}-\frac{\ell^{j} \cdot v^{k j} \cdot c^{j k}}{\ell^{k}}\left(j \in R^{k}(\bar{x})\right) .(
$$

Substituting the obtained values of $\alpha^{j}\left(j \in R^{k}(\bar{x})\right)$ into the first $r$ equalities $\left(r=\left|R^{k}(\bar{x})\right|\right)$ of (24) we get (22).

Lemma is proved.

Theorem 1. Let strictly positive vector $P=$ $\left(P^{1}, \ldots, P^{n}\right)$, index $k \in I$ и and a number $\mu^{k}$ defined by the formula (22) be given. The vector $\bar{x}$ is a solution of the $k-$ th consumer problem (8), satisfying the relation

$$
I_{1}(\bar{x})=\emptyset
$$

If and only if when

$$
\begin{gathered}
\left\{\begin{array}{l}
\ell^{i} \cdot v^{k i}=\mu^{k} \cdot P^{i} \forall i \in Q^{k}(\bar{x}), \\
\ell^{j} \cdot v^{k j}=\mu^{k} \cdot P^{j} \forall j \in R^{k}(\bar{x}) ;
\end{array}\right. \\
P \in \frac{1}{\mu^{k}} \cdot\left(\ell_{v}^{k}+\partial \tilde{q}^{k}\right),
\end{gathered}
$$

when $\ell_{v}^{k}, \partial \tilde{q}^{k}$ are defined in the lemma 3 .

Proof. Necessity. Let $\bar{x}$ is a solution of the problem (8), satisfying the relation (18). From lemmas 2 and 3 we get that there exists $\mu^{k}>0$, such that $\mu^{k} \cdot P \in \ell_{v}^{k}+\partial q^{k}$ i.e. (22) is satisfied. Then using the proof of lemma 4 (namely formula (25))

$$
\alpha^{j}=\mu^{k} \cdot \frac{P^{j} \cdot c^{j k}}{\ell^{k}}-\frac{\ell^{j} \cdot v^{k j} \cdot c^{j k}}{\ell^{k}},
$$

the condition $\alpha^{j} \geq 0$ for all $j \in R^{k}(\bar{x})$ and formula (22) we get the system of inequalities in the system (26). The first system of equalities of (26) follows from (23).

Sufficiency. Let the conditions (26) and (27) take place. Let us choose $\alpha^{j}\left(j \in R^{k}(\bar{x})\right)$ by the formula (25) which due (26) satisfies to the relations $\alpha^{j} \geq 0$, moreover $\sum_{j \in R^{k}(\bar{x})} \alpha^{j}=$ 1. Then as follows from the lemmas 2, 4 and (27) the number $\mu^{k}$ has a form (22) and $q^{k}(g) \leq 0 \forall g \in \Omega$, which indeed is a necessary and sufficient condition for optimality of $\bar{x}$ in the $k$-th branch.

Theorem is proved.

Remark. If $R^{k}(\bar{x})=I \equiv\{1,2, \ldots, n\}$ then the number $\mu^{k}$ defined by the formula (22) is the maximum growth rate of the total wealth of the $k$-th branch.

2. Consider the case when

$$
I_{1}(\bar{x}) \neq \varnothing \text {.- }
$$

Then according to Lemma $1 I_{1}(\bar{x}) \subset R^{k}(\bar{x})$.

Introduce the projection operator $P_{r_{m}}$ taking for $g=$ $\left(g^{1}, \ldots, g^{n}\right) \in R^{n}$

$$
P_{r_{m}}(g)=\left(g^{1}, \ldots, g^{m-1}, g^{m+1}, g^{n}\right) .
$$

Consider the vectors $L_{m}^{k} \in R^{n-1}$ and functions $\tilde{q}_{m}^{k}, q_{m}^{k}(m, k \in I)$ defined on $R^{n-1}$ as below

$$
\begin{gathered}
L_{m}^{k}=\left(\frac{\ell^{1}}{P^{1}} v^{k 1}-\frac{\ell^{m}}{P^{m}} v^{k m}\right) \\
\cdot P^{1}, \ldots,\left(\frac{\ell^{m-1}}{P^{m-1}} v^{k m-1}-\frac{\ell^{m}}{P^{m}} v^{k m}\right) \cdot P^{m-1}, \\
\left(\frac{\ell^{m+1}}{P^{m+1}} v^{k m+1}-\frac{\ell^{m}}{P^{m}} v^{k m}\right) \cdot P^{m+1}, \ldots,\left(\frac{\ell^{n}}{P^{n}} v^{k n}-\frac{\ell^{m}}{P^{m}} v^{k m}\right) \\
\cdot P^{n}, \\
\left\{\begin{array}{c}
\ell^{k} \cdot \min _{i \in R^{k}(\bar{x})} \frac{h^{i}}{c^{i k}}, m \in Q^{k}(\bar{x}) ; \\
\ell^{k} \cdot \min _{i \in R^{k}(\bar{x}) \backslash\{m\}}\left\{\frac{h^{i}}{c^{i k}},-\frac{1}{p^{m} \cdot c^{m k}} \cdot \sum_{i \in I \backslash\{m\}} P^{j} \cdot h^{j}\right\}, m \in R^{k}(\bar{x}) ; \\
q_{m}^{k}(h)=\left[L_{m}^{k}, h\right]+\tilde{q}_{m}^{k}(h) .
\end{array}\right.
\end{gathered}
$$

Note that the functions $q_{m}^{k}, \tilde{q}_{m}^{k}$ are superlinear. Take

$$
I_{1}^{m}=I_{1}(\bar{x}) \backslash\{m\}, T_{m}=\left\{\tilde{g} \in R^{n-1} \mid g^{i} \geq 0, i \in I_{1}^{m}\right\} .
$$

The cone $T_{m}^{*}$ adjoint to the cone $T_{m}$ has a form

$$
T_{m}^{*}=\left\{w \in R_{+}^{n-1} \mid w^{i} \geq 0, i \in I_{1}^{m} ; w^{i}=0, i \notin I_{1}^{m}\right\} .
$$

We'll consider the functional $q_{m}^{k}(\tilde{g})$ only on the cone $T$. Proposition 1 . For any $m \in I$ the conditions

1. $q^{k}(g) \leq 0$ for all $g \in G_{\bar{x}}(V)$;

2. $q_{m}^{k}(\tilde{g}) \leq 0$ for all $\tilde{g} \in T_{m}$; are equivalent.

Proof. Let $q^{k}(g) \leq 0\left(g \in G_{\bar{x}}(V)\right)$ i.e. (see (15))

$\left[\ell_{v}^{k}, g\right]+\ell^{k} \cdot \min _{i \in R^{k}(\bar{x})} \frac{g^{i}}{c^{i k}} \leq 0, g \in G_{\bar{x}}(V)$.

Due to (13) e have $[P, g]=0$; fixing the index $m \in I$, expressing $g^{m}$ and substituting into the left hand side on the last inequality, after some eliminations we get (31).

Having the same argument in reverse order, we get the contradiction.

Proposition is proved.

In the case when $I_{1}(\bar{x}) \neq \varnothing$ the subdifferential $\partial q_{m}^{k}$ has a form $[8,10]$

$$
\partial q_{m}^{k}=L_{m}^{k}+\partial \tilde{q}_{m}^{k}+T_{m}^{*}(k, m \in I),
$$

where $L_{m}^{k}, \partial \tilde{q}_{m}^{k}, T_{m}^{*}$ are defined in the formulas (28), (30) [2, 3].

Theorem 2. Let the strictly positive vector $P=$ $\left(P^{1}, \ldots, P^{n}\right)$ be given. If the vector $\bar{x}$ for which

$$
I_{1}(\bar{x})=\emptyset,
$$

is a maximum point in the $k$-th consumer problem (8), then for $m \in Q^{k}(\bar{x})$ the following relation is satisfied 


$$
\left\{\begin{array}{l}
\frac{\ell^{i}}{P^{i}} \cdot v^{k i} \leq \frac{\ell^{m}}{P^{m}} \cdot v^{k m} \forall i \in R^{k}(\bar{x}), \\
\frac{\ell^{j}}{P^{j}} \cdot v^{k j}=\frac{\ell^{m}}{P^{m}} \cdot v^{k m} \forall j \in Q^{k}(\bar{x}) ;
\end{array}\right.
$$

for $m \in R^{k}(\bar{x})$ is satisfied:

$$
\left\{\begin{array}{c}
\frac{\ell^{i}}{P^{i}} \cdot v^{k i} \leq \frac{\ell^{j}}{P^{j}} \cdot v^{k j} \forall i \in R^{k}(\bar{x}), j \in Q^{k}(\bar{x}) \\
\frac{\ell^{i}}{P^{i}} \cdot v^{k i}=\frac{\ell^{m}}{P^{m}} \cdot v^{k m} \forall i \in R^{k}(\bar{x}) ;
\end{array}\right.
$$

2) Let $I_{1}(\bar{x}) \neq \varnothing$ and for some $m$ is valid (34) (if $m \in$ $Q^{k}(\bar{x})$ ) or (35) (if $m \in R^{k}(\bar{x})$ ). Then the vector $\bar{x}$ is a solution of the $k$-th consumer problem (8).

Proof. Necessity. Let strictly positive vector $P=\left(P^{1}, \ldots, P^{n}\right)$ be given and $\bar{x}$ is the point at which the utility function of the $\mathrm{k}$ th branch of (8) satisfying (33) takes its maximum.

Then due the proposition 1 necessary and sufficient optimality conditions for the vector $\bar{x}$ in the $k$-th branch take the form

$$
q_{m}^{k}(\tilde{g}) \leq 0 \forall \tilde{g} \in T_{m}(m, k \in I),
$$

where the cone $T_{m}$ is defined by the formula (29).

By the definition of the superdifferential we have

$$
q_{m}^{k}(\tilde{g}) \leq 0 \forall \tilde{g} \in t_{m} \Leftrightarrow 0 \in \partial q_{m}^{k}(k \in I),
$$

where superdiffeential $\partial q_{m}^{k}$ has a form (32).

Consequently

$$
-L_{m}^{k} \in \partial \tilde{q}_{m}^{k}+T_{m}^{*}(m, k \in I) .
$$

Depending on the choice of the index $m \in I$ the function $\tilde{q}_{m}^{k}(g)$ may have various forms (see (28)).

1) Let $m \in Q^{k}(\bar{x})$. Then from (28) we have

$$
\tilde{q}_{m}^{k}(g)=\ell^{k} \cdot \min _{i \in R^{k}(\bar{x})} \frac{g^{i}}{c^{i k}} .
$$

Superdifferential of this function has form (21).

Substituting (21), (30) and (28) into the relation (36) we get that there exist the numbers $\alpha^{i} \geq 0, w^{i} \geq 0$ such that

$$
\begin{gathered}
\sum_{i \in R^{k}(\bar{x})} \alpha^{i}=1, \\
-\frac{\alpha^{i}}{c^{i k}} \cdot \ell^{k}-w^{i}=P^{i} \cdot\left(\frac{\ell^{i}}{P^{i}} v^{k i}-\frac{\ell^{m}}{P^{m}} v^{k m}\right) \forall i \in R^{k}(\bar{x}), \\
0=P^{j} \cdot\left(\frac{\ell^{j}}{P^{j}} v^{k j}-\frac{\ell^{m}}{P^{m}} v^{k m}\right) \forall j \in Q^{k}(\bar{x}) .
\end{gathered}
$$

From this immediately follows the inequality (34).

We claim the opposite. Suppose that the conditions of the theorem hold true in the case when $m \in Q^{k}(\bar{x})$, i.e. we can choose the numbers $\alpha^{i} \geq 0$ and $w^{i} \geq 0\left(i \in R^{k}(\bar{x})\right)$ by such way that the inequality (37) would be satisfied and $\sum_{i \in R^{k}(\bar{x})} \alpha^{i}=1$. It means that the relation (36) is satisfied or $0 \in \partial q_{m}^{k}$. Consequently, due to the definition of the superdifferential the following condition takes place $q_{m}^{k}(\tilde{g}) \leq 0 \forall \tilde{g} \in T_{m}\left(m \in Q^{k}(\bar{x})\right)$, that indeed is necessary and sufficient condition for the optimality of the vector $\bar{x}$ in the $k$-th branch.

2) Consider the case when $m \in R^{k}(\bar{x})$.

Then from (28) we get

$$
q_{m}^{k}(\tilde{g})=\ell^{k} \cdot \min _{i \in R^{k}(\tilde{x}) \backslash\{m\}}\left\{\frac{g^{i}}{c^{i k}},-\frac{1}{P^{m} \cdot c^{m k}} \cdot \sum_{i \in I \backslash\{m\}} P^{i} \cdot g^{i}\right\},
$$

Superdifferential of which has a form $[8,9]$

$$
\begin{aligned}
& \partial \tilde{q}_{m}^{k}=\ell^{k} \cdot \operatorname{CO}\left\{\frac{1}{c^{i k}} \cdot e^{i}, \widetilde{\ell} \mid i \in R^{k}(\bar{x}) \backslash\{m\},\right. \\
& \begin{array}{c}
\left.\tilde{\ell}=-\frac{1}{P^{m} \cdot c^{m k}} \cdot\left(P^{1}, \ldots, P^{m-1}, P^{m+1}, \ldots, P^{n}\right)\right\} \\
=\left\{f \in R^{n-1} \mid f=\ell^{k} .\right.
\end{array} \\
& \left(\sum_{i \in R^{k}(\bar{x}) \backslash\{m\}} \alpha^{i} \cdot \frac{1}{c^{i k}} \cdot e^{i}-\frac{\alpha^{m}}{P^{m \cdot c^{m k}}} \cdot\left(P^{1}, \ldots, P^{m-1}, P^{m+1}, \ldots, P^{n}\right)\right), \\
& \left.\alpha^{i} \geq 0, \sum_{i \in R^{k}(\bar{x})} \alpha^{i}=1\right\} \\
& =\left\{f=\left(f^{1}, \ldots, f^{n-1}\right) \in R^{n-1} \mid \exists \alpha^{i}\right. \\
& \geq 0: \sum_{i \in R^{k}(\bar{x})} \alpha^{i}=1 \\
& f^{i}=\left(\frac{\alpha^{i}}{c^{i k}}-\frac{\alpha^{m}}{P^{m} \cdot c^{m k}} \cdot P^{i}\right) \ell^{k}, i \in R^{k}(\bar{x}) ; f^{i} \\
& \left.=-\frac{\alpha^{m}}{P^{m} \cdot c^{m k}} \cdot P^{i} \cdot \ell^{k}, i \in Q^{k}(\bar{x})\right\} \text {. }
\end{aligned}
$$

The according to (28), (30) and (38) from (36) follows that there exists numbers $\alpha^{i} \geq 0, w^{i} \geq 0\left(i \in R^{k}(\bar{x})\right)$ such that

$$
\begin{gathered}
\sum_{i \in R^{k}(\bar{x})} \alpha^{i}=1, \\
\left(\frac{\alpha^{m}}{P^{m} \cdot c^{m k}} \cdot P^{i}-\frac{\alpha^{i}}{c^{i k}}\right) \cdot \ell^{k}-v^{i} \\
=\left(\frac{\ell^{i}}{P^{i}} v^{k i}-\frac{\ell^{m}}{P^{m}} v^{k m}\right) \cdot P^{i} \forall i \in R^{k}(\bar{x}), \\
\frac{P^{i} \ell^{k}}{P^{m} c^{m k}} \cdot \alpha^{m}=\left(\frac{\ell^{j}}{P^{j}} v^{k j}-\frac{\ell^{m}}{P^{m}} v^{k m}\right) \cdot P^{j} \forall j \in Q^{k}(\bar{x}) .
\end{gathered}
$$

These lead us to

$$
\begin{aligned}
\frac{\ell^{i}}{P^{i}} v^{k i} & \leq \frac{\ell^{m}}{P^{m}} v^{k m} \forall i \in R^{k}(\bar{x}), \\
\frac{\ell^{j}}{P^{j}} v^{k j} & \geq \frac{\ell^{m}}{P^{m}} v^{k m} \forall j \in Q^{k}(\bar{x}) .
\end{aligned}
$$

Since the first inequality of the last system holds true for $\forall i, m \in R^{k}(\bar{x})$, it turns to the equality. As a result, we obtain the desired result.

Let's claim the opposite. Suppose that the conditions of the theorem in the case when $m \in R^{k}(\bar{x})$, i.e. we can choose the 
numbers $\alpha^{i} \geq 0$ and $w^{i} \geq 0\left(i \in R^{k}(\bar{x})\right)$ such that the inequality (39) would be satisfied or $0 \in \partial q_{m}^{k}$. This is equivalent to the condition $q_{m}^{k}(\tilde{g}) \leq 0 \forall \tilde{g} \in T_{m}$, that is indeed a necessary and sufficient condition for the optimality of the vector $\bar{x}$ in the $k$-th branch. Theorem is proved.

\section{Conclusion}

(1). The form of the superdifferential of the utility function is defined.

(2). Necessary and sufficient condition for the existence of the maximum of this function is derived.

(3). The maximum rate of the growth of the industries total wealth determined.

(4). A necessary and sufficient condition is obtained or optimality of the state vector of the branches.

\section{References}

[1] Makarov V. L., Rubinov A. M. The mathematical Theory of Economic Dynamics and Equilibrium, Moscow, Nauka, 1973, $336 \mathrm{p}$.
[2] Crassus I. A. Models of Economic Dynamics, Moskow, Sov. Radio, 1976, 278 p.

[3] Lancaster K. Mathematical Economics, Moskow, Sov Radio, 1972.

[4] Rubinov A. M. On a Leontief type model, Optimization, 1962, 32 (49), pp 109-127.

[5] Dobrescu L. I., Neamtu M., Ciurdariu A. L., Orpish D. A. Dinamic Economic Model with Discrete Time and Consumer Sentiment. Discrete Dynamics in Nature and Society, 2009, Article ID509561.

[6] Doms M. and Morin N., Consumer Sentiment, the Economy and the News Media, Federal Reserve Bank of San-Francisco, San-Francisco, California, USA, 2004.

[7] Westerhoff F. H. Consumer sentiment and business cycles a Neimark-Sacker bifurcation scenario, Applied Economics Letters, Vol. 15, no 15, 2008, pp. 1201-1205.

[8] Demyanov V. F., Vasilyev L. V. Non differentiable Optimization, Moskow, Nauka, 1981, 384 p.

[9] Demyanov V. F., Rubinov A. M. Approximate Methods of Solving Extremal Problems, LSU, 1968, 180 p.

[10] Rockafellar R. Convex Analysis, Moskow, 1973, 471 p. 